\title{
The Solar Origins of the Sun-Earth Connection Events on April 1999 and February 2000
}

\author{
L. A. Balmaceda ${ }^{1,2}$, W. D. Gonzalez ${ }^{1}$, E. Echer ${ }^{1}$, J. C. Santos ${ }^{1}$,L. E. A. Vieira ${ }^{1}$, \\ A. Dal Lago ${ }^{1}$, F. L. Guarnieri ${ }^{1}$, and A. L. C. Gonzalez ${ }^{1}$ \\ ${ }^{1}$ Instituto Nacional de Pesquisas Espaciais, INPE - S. José dos Campos, SP, Brazil \\ ${ }^{2}$ Max-Planck-Institut fuer Sonnensystemforschung, MPS, Katlenburg-Lindau, Germany
}

Received on 2 February, 2004; revised version received on 6 May, 2004

\begin{abstract}
In this work we present a comparison between the solar origins of two solar-terrestrial connection events occurring on April 1999 and February 2000, respectively. On April 13 (1999), a coronal mass ejection (CME) appeared for the first time in LASCO-C2 coronagraph field of view at 03:30 UT. The possible solar disc source of this CME was located at coordinates N25 E05, and it consisted on flaring activity together with a filament eruption as seen in EIT images. The geomagnetic storm with a Dst peak of $-91 \mathrm{nT}$ registered on April, 17 was attributed to this solar surface activity. On February 8, 9 and 10 (2000) three Earth-directed halo CMEs were recorded by LASCO coronagraphs. The interaction of two of them caused the intense interplanetary geomagnetic storm registered on February 12 (Dst $=-110 \mathrm{nT}$ ). The CMEs detected on February 8 and 10 were launched from AR 8858 and the source of the activity was attributed to two solar flares of X-ray class M1.3 and C7.3 respectively. The CME detected on February 9 was originated in AR 8853 and flaring activity was also identified as the solar source for this event. In this study we discuss the solar origins of the events that gave rise to coronal and interplanetary disturbances and as a consequence produced geomagnetic activity at the Earth. This work is part of a complete study that is searching the solar origin, the interplanetary aspects and the magnetospheric effects of these events.
\end{abstract}

\section{Introduction}

CMEs directed along the Sun-Earth line can be detected as halos around the occulting disk in a white-light coronagraph $[1,2]$. It is important to distinguish between a full halo that extends $360^{\circ}$ around the Sun and a partial halo . An event is classified as a partial halo if the apparent width is greater than $120^{\circ}$ and if it appears in projection above at least one of the poles; this latter criterion is an attempt to exclude limb events that just happen to be large but do not have a component along a line between the Sun and Earth. The full and partial halos represent density enhancements propagating over a wide range of angles, presumably as a result of material moving near the Sun-Earth line. From changes in the halo pattern, one can estimate speeds in the plane of the sky. Without knowledge of the true angular extent of a mass ejection, it is difficult to know its speed along the Sun-Earth line. Hence, any quantitative comparison of these projected speeds for halo CMEs should be considered speculative and used with caution in the interpretation of ejecta at 1 AU. In this work we examined the white-light images of the corona obtained by the Large Angle and Spectrometric Coronagraph (LASCO) [3] on board the Solar and Heliospheric Observatory (SOHO) mission to identify the solar causes of geomagnetic activity. For this reason, we considered only earthward directed CMEs, as determined from signatures in the Extreme ultraviolet Imaging Telescope (EIT) data [4] and from observations of associated disappearing filaments and flares in $\mathrm{H}$-alpha line and other wavelenghts. In the following section we present the description of the solar disc sources of the CMEs that occurred on April, 1999 and February 2000 and that caused two geomagnetic storms.

\section{Description of the events}

The moderate geomagnetic storm registered on April 17, 1999 was caused by a single CME while a more intense geomagnetic activity detected on February 12, 2000 was attributed to the complex ejecta resulting of the interaction of CMEs in the interplanetary medium. In Table 1 we present the time appearing in LASCO-C2 field of view, the planeof-sky velocity, the onset time estimated from considering a constant velocity, the angular width and the central position angle (PA) measured from solar north (counter clockwise) of the single CME occurred on April 13, 1999 and the three CMEs occurred on February 8, 9 and 10, 2000.

\subsection{April 13, 1999 event}

On April 13, 1999 a halo CME directed to Earth was observed appearing for the first time in LASCO C2 field of view at 03:30 UT. This CME seems to be associated to the flaring activity observed at solar surface. In the active region located at heliographic coordinates N20 E02 a two ribbon flare and ejecta material occurred according to [5] as it can be seen in Fig. 1. The estimated onset time obtained by assuming a constant speed is at 01:44 UT. 
TABLE 1. Dynamical and morphological properties of CMEs.

\begin{tabular}{lccccc}
\hline CME date & $\begin{array}{c}\text { Time } \\
\text { LASCO-C2 } \\
{[\mathrm{UT}]}\end{array}$ & $\begin{array}{c}\text { Velocity } \\
{[\mathrm{km} / \mathrm{s}]}\end{array}$ & $\begin{array}{c}\text { Onset } \\
\text { Time } \\
{[\mathrm{UT}]}\end{array}$ & $\begin{array}{c}\text { Angular } \\
\text { Witdh } \\
{\left[{ }^{\circ}\right]}\end{array}$ & $\begin{array}{c}\text { Central } \\
\text { Position } \\
\text { Angle }\left[{ }^{\circ}\right]\end{array}$ \\
\hline April 13, 1999 & $03: 30$ & 291.2 & $01: 44$ & $>86$ & 194 \\
\hline February 8, 2000 & $09: 30$ & 1079.5 & $08: 51$ & 360 & 50 \\
\hline February 9, 2000 & $19: 54$ & 910.0 & $19: 14$ & 360 & 223 \\
\hline February 10, 2000 & $02: 30$ & 943.7 & $01: 38$ & 360 & 340 \\
\hline
\end{tabular}
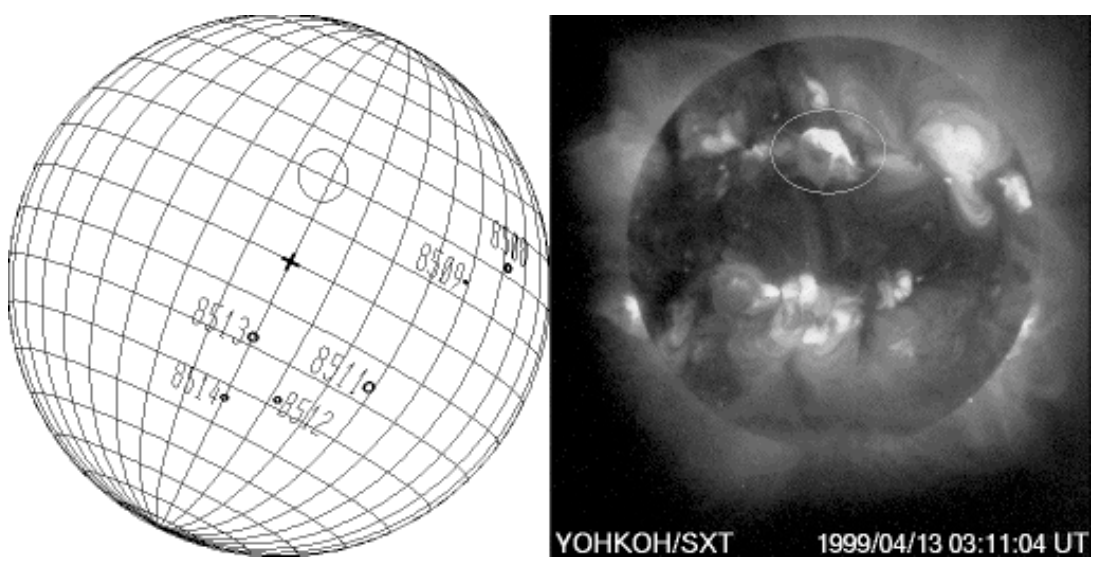

Figure 1. Flaring activity was observed in X-rays solar images from Yohkoh satellite (right) on April 13, 1999. In the active region located at heliographic coordinates N20 E02 (left) a two ribbon flare and ejecta material occurred.

\subsection{February 8, 9 and 10, 2000 events}

In Fig. 2 three successive CMEs observed by LASCO coronagraphs occurring on February 8, 9 and 10 are shown. On February 8, 2000 a M1.3 X-ray flare was detected by GOES 8 satellite. This flare started at 08:42 UT, finishing at 09:18 UT and it occurred in active region 8858 located at heliographic coordinates N25 E26. In H-alpha images a flare of importance 1B was observed to occur between 08:43 and 09:59 UT. A coronal wave was also observed by EIT, beginning at 08:48 UT. At 09:30 UT a CME related to this solar surface activity was recorded by LASCO C2 appearing as a bright, structured loop above the NE limb. It was later visible at all position angles, although it was very faint in the SW. The plane-of-sky speed measured at PA 50 (NE limb) was $1079.5 \mathrm{~km} / \mathrm{s}$. On February 9, 2000 active region 8853 presented flaring activity observed in H-alpha. Starting at 19:26 UT a solar flare with intensity $2 \mathrm{f}$ was detected at heliographic coordinates S17 W40. Also an X-ray flare class C7.4 was reported by GOES satellite occurring in the same region. This solar surface activity ended at 21:34 UT. EIT observed this event as a filament activation over several hours, with eruption of the filament at 19:25 UT. Bright post-eruption loops were observed beginning at 19:48 UT. LASCO-C2 detected a CME halo-type appearing in the field of view at 19:54 UT. The event was observed first in the $\mathrm{SW}$, and later extended all around the occulting disk. The leading edge appeared as a fuzzy front followed by a bright sharp edge. The plane-of-sky speed was $910 \mathrm{~km} / \mathrm{s}$. This speed was measured at PA 223 (SW limb) corresponding to the central position of the CME. On February 10, a X-ray solar flare class C7.3 occur in active region AR 8858. The heliographic position of this flare was N31 E04 and it started at 01:40 UT lasting until 02:39 UT. EIT observed a flare and coronal wave from this region beginning at about 01:25 UT. The CME was observed in LASCO C2 field of view at 02:30 UT over de N pole and later extended over all position angles. The plane-of-sky speed at PA 340 (NW limb) was $943.7 \mathrm{~km} / \mathrm{s}$. By analyzing interplanetary data, it is observed that the geomagnetic activity registered on February 12, 2000 was caused by the interaction of CMEs. Although it is complicated to correctly identify which ones of these three events are involved, we propose that is more likely that CMEs occurring on 9 and 10 are the ones that interact based on the following considerations. First of all, it is important to compare the velocities for each event since the preceeding CME must be necessarily slower than the subsequent to be reached by it facilitating the interaction. Thus, we observe that this condition is only fullfilled by the CMEs that occurred on February 9 and 10. Since we know the heliographic coordinates of the solar disc sources, we can infer the separation between the two CMEs that possibly interact. Thus, we found a separation of $42^{\circ}$ in latitude and $66^{\circ}$ in longitude for the CMEs occurring on February 8 and 9 , 


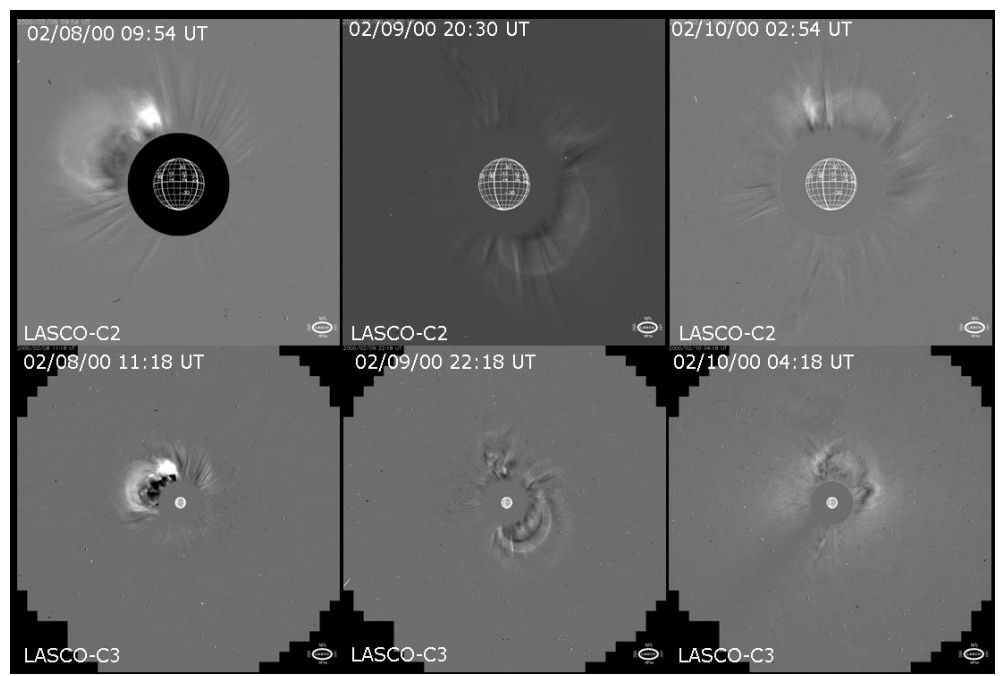

Figure 2. On February 8 a CME was recorded by LASCO C2 appearing above the NE limb (top left) at 09:30 UT. On February 9 a CME halo-type appeared at 19:54 UT (top middle). On February 10 a CME was observed at 02:30 UT over de N pole (top right). The three images below correspond to each CME detected by LASCO-C3 coronagraph.

while the separation between the position of the solar sources of February 9 and 10 were $48^{\circ}$ in latitude and $44^{\circ}$ in longitude. Note that this separation is smaller than in the previous case. This is also consistent with the separation between the central position angles of the two pairs of CMEs which were $173^{\circ}$ and $117^{\circ}$ respectively. This separation is also smaller than the angular width of February 10, $2000 \mathrm{CME}$ making possible a physical overlap between this one and the February 9, 2000 CME. Another aspect to take into account to correctly determine the CME interaction is the time difference between each candidate. In this way, the CME occurring on day 9 appears in LASCO-C2 field of view aproximately 34 hours after the first one while the time difference between this one and the CME observed on 10 is smaller being only 6 hours.

\section{Final Remarks}

In this work we have investigated the solar sources of two geomagnetic storms that occurred on April 17, 1999 and February 12,2000 . The main difference between both events is that the first one was caused by a single CME detected on April 13, 1999 while the second one was caused by the interaction of two CMEs observed on February 9 and 10, 2000. Using solar wind observations at $1 \mathrm{AU}$, we can distinguish these events into two classes of fast ejecta: the first one identified as magnetic cloud [6], whose local magnetic structure is that of a flux rope and the second one identified as complex ejecta, which are not magnetic clouds and have disordered magnetic fields resulting from the interaction between CMEs. CMEs are large-scale magnetic structures carrying ionized plasma, for this reason the physics of the interaction is very complex. Some authors speculate that the interaction between two CMEs may result in reconnection or simple piling up of the slower CME at the leading edge of the faster CME, resulting in a complex structure. Beyond the interaction region, one would expect a single CME consisting of magnetic field lines and plasma from both CMEs. Many authors called this process as CME cannibalism [7]. The final structure and composition of the resulting CME depend upon the nature of the interaction between the two CMEs. Although this kind of phenomena was studied by other authors the nature of the interactions is still not understood, and the results of such interactions have not been predicted.

\section{References}

[1] R. A. Howard, D. J. Michels, N. R. Sheeley, and M. J. Koomen, Astrophysical Journal, Part 2 - Letters to the Editor, 263, (1982).

[2] S. P. Plunkett, B. J. Thompson, R. A. Howard, D. J. Michels, O. C. St. Cyr, S. J. Tappin, R. Schwenn, and P. L. Lamy, Geophysical Research Letters 25, 14 (1998).

[3] G. E. Brueckner, R. A Howard, M. J. Koomen, C. M. Korendyke, D. J. Michels, J. D. Moses, D. G. Socker, K. P. Dere, P. L. Lamy, A. Llebaria, M. V. Bout, R. Schwenn, G. M. Simnett, D. K. Bedford, and C. J. Eyles, Solar Physics, 162, (1995).

[4] J.-P. Delaboudiniere, G. E. Artzner, J. Brunaud, A. H. Gabriel, J. F. Hochedez, F. Millier, X. Y. Song, K. P. Dere, et al., Solar Physics, 162, (1995).

[5] G. Zhou, J. Wang, and Z. Cao, Astronomy and Astrophysics, 397, (2003).

[6] L. F. Burlaga, K. W. Behannon, Solar Physics, 81, (1981).

[7] N. Gopalswamy, S. Yashiro, M. L. Kaiser, R. A. Howard, and J.-L. Bougeret, The Astrophysical Journal, 548, (2001). 